\title{
Inflação Implícita e o Prêmio pelo Risco: Uma Alternativa aos Modelos VAR na Previsão para o IPCA
}

\author{
João F. Caldeira \\ Professor - PPGA e PPGE - Universidade Federal do Rio Grande do Sul (UFRGS) \\ Endereço para contato: Av. João Pessoa, 52 - sala 33 B - $3^{\circ}$ andar, Centro - Porto Alegre/ RS \\ Brasil - CEP: 90040-000 - E-mail: joao.caldeira@ufrgs.br
}

\section{Luiz G. C. Furlani}

Analista de Risco de Mercado Sênior do Banco Cooperativo Sicredi SA e PPGE - Universidade Federal do Rio Grande do Sul (UFRGS) - Endereço para contato: Av. Assis Brasil, 3940 - Torre C, $11^{\circ}$ andar - Porto Alegre/ RS - Brasil - CEP 91060-900 - E-mail: luiz_furlani@sicredi.com.br

Recebido em 14 de agosto de 2012. Aceito em 28 de junho de 2013.

\section{Resumo}

O presente artigo avalia, para o caso brasileiro, se a inflação implícita extraída dos títulos de renda fixa constitui um estimador não viesado da inflação ao consumidor, medida pelo IPCA. Nossas estimativas sugerem que as break-even inflation rates ou simplesmente BEIRs - trazem informação relevante a respeito da inflação futura, especialmente para a maturidade de três meses. A principal inovação de nosso trabalho, contudo, está no método utilizado para a estimação, que nos permite concluir que o prêmio de risco da inflação, para algumas das maturidades consideradas, varia ao longo do tempo e não é desprezível do ponto de vista econômico. Comparamos também as previsões de inflação obtidas a partir das BEIRs com os modelos VAR utilizados pelo Banco Central e as estimativas dos Top5 do Relatório Focus. As previsões realizadas com as BEIRs mostraram maior acurácia que aquelas extraídas dos modelos VAR, porém, menos precisas que as geradas pelos Top5.

\section{Palavras-Chave}

break-even inflation rate, previsão de inflação, prêmio de risco de inflação

\begin{abstract}
The present paper examines, for the Brazilian case, if implied inflation extracted from fixed income securities is an unbiased estimator of consumer inflation, measured by the IPCA. Our estimates suggest that break-even inflation rates - or just BEIRs - are informative about future inflation, especially for the maturity of three months. The main innovation of our work, however, is the method used for estimation, allowing us to conclude that inflation risk, for some of the maturities considered, varies over time and is not irrelevant from the economic standpoint. We also compared the inflation forecasts obtained from BEIRs with the ones extracted from VAR models used by Central Bank and estimates of Focus Report's Top5s. The forecasts performed with BEIRs showed greater accuracy than those extracted from VAR models, though less precision than those generated by the Top5s.
\end{abstract}




\section{Keywords}

break-even inflation rate, inflation forecasting, inflation risk premium

\section{JEL Classification}

E31, E37, F41

\section{Introdução}

A ancoragem das expectativas de inflação é fundamental para a condução da política monetária e manutenção da estabilidade de preços na economia, especialmente para um Banco Central que segue um regime de metas para a inflação. Por esse motivo, as autoridades monetárias ao redor do mundo buscam, de forma incessante, indicadores confiáveis e apropriados para monitorar as expectativas de inflação dos agentes do mercado. Uma das maneiras de monitorar essas expectativas é através de títulos cuja rentabilidade está vincula à variação dos preços.

O uso de tais títulos para o referido propósito pode ser facilmente justificado: dada a sua característica forward-looking, além de um prêmio pelo risco incorrido, estes títulos também incorporam as expectativas dos investidores sobre a inflação futura. Autoridades do Banco Central Europeu - doravante ECB - e do Banco Central dos Estados Unidos - Federal Reserve, ou simplesmente Fed - consideram que as expectativas de inflação extraídas dos títulos acima mencionados configuram uma das mais importantes fontes para monitoramento das expectativas dos agentes de mercado, com a grande vantagem de permitir seu acompanhamento em tempo real. ${ }^{l}$

Bernanke (2004), atual presidente do Federal Reserve, afirma que uma das mais importantes fontes para as expectativas de inflação do mercado se encontra nos títulos com rentabilidade vinculada à

\footnotetext{
1 No caso do Fed são, basicamente, duas alternativas aos títulos vinculados à variação dos preços para o monitoramento das expectativas de inflação: Survey of Professional Forecasters e Consensus Economics. A primeira destas pesquisas, realizada pelo Fed da Filadélfia, tem divulgação trimestral, e a segunda, de divulgação mensal, é de responsabilidade de empresa privada, acessível somente aos assinantes do serviço. Desta forma, tais pesquisas falham ao não permitir um acompanhamento em tempo real das expectativas. O Consensus Economics também divulga expectativas para os países da Zona do Euro, porém, estas apresentam o mesmo problema: periodicidade muito baixa de divulgação e somente para assinantes.
} 
inflação, essencialmente TIPS. ${ }^{2}$ Garcia e van Rixtel (2007), do ECB, também defendem o uso de tais títulos para o monitoramento das expectativas de inflação dos participantes do mercado, assim como Hetzel (1992), Breedon (1995), Deacon e Andrews (1996), Barr e Campbell (1997), Kitamura (1997), Emmons (2000), entre outros.

Grishenko et al. (2007) seguem a mesma linha de Ang, Bekaert, e Wei (2008). Ambos usam os preços de mercado das TIPS para estimar o prêmio de risco da inflação. Campbell e Shiller (1996), Buraschi e Jiltsov (2005) e Haubrich et al. (2008) estimam o prêmio de risco da inflação sem utilizar as informações do mercado de TIPS. Campbel e Shiller (1996) realizam a estimação com base no prêmio a termo nominal. Já Buraschi e Jiltsov (2005) analisam o prêmio de risco nominal e real da estrutura a termo dos Treasuries norte-americanos, baseado na versão monetária estrutural do ciclo de negócios.

D’Amico, Kim e Wei (2006) estimam a estrutura a termo nominal com dados de Treasuries e dados de TIPS através de um modelo com três fatores, concluindo que os yields das TIPS contêm um componente substancial de liquidez. As medidas de longo prazo do prêmio de risco da inflação estimada em seu estudo assumem valores negativos e positivos. Jarrow e Yildirim (2003), Liu e Cheng (2005) também usam dados de TIPS para estimar modelos da estrutura a termo, mas não focam na estimação do prêmio de risco de inflação. Hordahl, Tristani e Vestin (2005) estimam o prêmio de risco de inflação da Zona do Euro, usando informações de taxas de juros nominais e yields de títulos indexados a índices de inflação. Evans et al. (1998) empregam uma condição de equilíbrio de primeira ordem para estruturas a termo nominal e real, utilizando-a para testar a hipótese de Fisher nos dados do Reino Unido.

Para o Brasil, destaque para o trabalho de Vicente e Guillen (2010). Neste estudo, os autores analisam se os títulos vinculados à variação dos preços contêm informação sobre a inflação futura. Para tanto, regridem a inflação acumulada no período contra o spread entre rendimentos de títulos nominais e reais - i.e., a expectativa de inflação implícita nestes títulos, denominada BEIR -, além de uma constante. Os dados apontam que a BEIR, além de informativa, é um estimador

2 TIPS é o acrônimo de Treasury Inflation-Protected Securities. Estes títulos possuem rentabilidade vinculada à variação do índice de preços ao consumidor norte-americano - o CPI -, e cupom semestral. 
não viesado para a inflação em horizontes de 3 e 6 meses. A BEIR também é informativa para 24 e 30 meses, porém, não apresenta poder explicativo para 12 e 18 meses. Segundo os autores, a falta de poder explicativo para horizontes intermediários pode ser explicada, entre outros fatores, pela magnitude da compensação exigida pelo risco, que, para o mercado brasileiro, pode ser significativa.

Este prêmio pelo risco, segundo Garcia e van Rixtel (2007), é resultante, basicamente, de dois fatores: o primeiro deles está associado a uma compensação pela incerteza relacionada à inflação futura, especialmente em títulos de maturidades mais longas, enquanto o segundo refere-se a uma compensação pela liquidez. Isto significa que, naturalmente, existe uma compensação pelo risco incorrido e que esta compensação, não necessariamente, é constante ao longo do tempo - ou maturidades -, como assumem Vicente e Guillen (2010).

O presente trabalho se propõe a avaliar, como objetivo primário, para o caso brasileiro, se as informações sobre a inflação implícita extraídas dos títulos de renda fixa refletem as expectativas dos agentes sobre a inflação futura, sem partir de hipóteses tão restritivas quanto às impostas por Vicente e Guillen (2010). Isto será realizado ao permitir que a compensação pelo risco e o poder explicativo da inflação implícita variem ao longo do tempo. $O$ trabalho também se dispõe a determinar o poder preditivo da BEIR frente a outras medidas de expectativas de inflação, tais como as constantes nos Relatórios Focus e as extraídas dos modelos autorregressivos (VAR), do Banco Central do Brasil (BCB).

O restante deste trabalho está organizado da seguinte forma: a seção 2 apresenta o modelo econométrico utilizado atender ao objetivo principal deste estudo; a seção 3 detalha os dados utilizados e o tratamento dado a eles; a seção 4 apresenta os resultados da estimação; na seção 5 , é realizada a avaliação do poder predito da BEIR frente às expectativas constantes nos Relatórios Focus e modelos VAR, do BCB; e, por fim, a seção 6 , apresenta as conclusões. 


\section{O Modelo}

O modelo que utilizamos para avaliar se as informações sobre a inflação implícita extraídas dos títulos de renda fixa, de fato, refletem as expectativas dos agentes sobre a inflação futura, é semelhante ao utilizado por Vicente e Guillen (2010). ${ }^{3}$ Seja $y_{t}^{N}(\tau)$ e $y_{t}^{R}(\tau)$ taxas nominais e reais, respectivamente, continuamente compostas, no tempo $t$ e com maturidade $\tau$. O spread entre os rendimentos de títulos nominais e reais - i.e., a expectativa de inflação implícita nestes títulos, denominada BEIR -, é dada por:

$$
i_{t}(\tau)=y_{t}^{N}(\tau)-y_{t}^{R}(\tau)
$$

A variação anualizada nos preços entre dois períodos - i.e., a taxa de inflação observada para o período -, entre $t$ e $t+\tau$, por exemplo, é dada por $h_{t}(\tau)$ :

$$
h_{t}(\tau)=\frac{1}{\tau} \sum_{j=t}^{t+\tau-1} h_{j}(\tau)
$$

A informação sobre a inflação implícita e seu poder explicativo sobre a inflação observada pode ser medido pela seguinte equação:

$$
h_{t}(\tau)=c_{1}^{\tau}+c_{2}^{\tau} i_{t}(\tau)+\varepsilon_{t}
$$

Vicente e Guillen (2010) utilizam a Equação (3) para testar se o spread entre rendimentos de títulos nominais e reais contém alguma informação sobre a inflação futura. Para que isto seja verdadeiro, $c_{2}^{\tau}$ deve ser diferente de zero. Para que este spread seja um estimador não viesado da inflação futura, é necessário que, conjuntamente, $c_{1}^{\tau}=0$ e $c_{2}^{\tau}=1$. Intuitivamente, é fácil verificar esta relação. Para tanto, basta reescrever a Equação (3) para a inflação futura, chegando à relação descrita na introdução deste trabalho: o spread entre rendimentos de títulos nominais e reais é igual à inflação futura esperada acrescida de uma compensação pelo risco.

\footnotetext{
3 Por simplificação, utilizamos, inclusive, as mesmas notações de Vicente e Guillen (2010).
} 
Contudo, conforme mencionado anteriormente, objetivamos estimar (3) sem impor qualquer tipo de restrição na compensação pelo risco incorrido, pois há evidências de que esta, não necessariamente, é constante ao longo do tempo, como sugerem Grishchenko e Huang (2010). Da mesma forma, o coeficiente associado à inflação implícita não necessariamente é constante e, assim, também permitiremos que ele varie ao longo do tempo. É necessário, então, estimar o seguinte sistema:

$$
\begin{aligned}
& h_{t}(\tau)=c_{1, t}^{\tau}+c_{2, t}^{\tau} i_{t}(\tau)+\varepsilon_{t} \\
& c_{1, t}^{\tau}=c_{1, t-1}^{\tau}+\eta_{t} \\
& c_{2, t}^{\tau}=c_{2, t-1}^{\tau}+\zeta_{t}
\end{aligned}
$$

Estimaremos o sistema descrito em (4) através do Filtro de Kalman. Para tanto, as Equações em (4) devem ser reescritas na representação de espaço de estado, divididas na Equação de medida (5) - que relaciona o vetor de observações com o vetor de estados, a variável explicativa e o erro de medida - e na Equação de transição (6) - que descreve uma Cadeia de Markov de primeira ordem para governar a transição do estado com inovações:

$$
\begin{aligned}
& {\left[h_{t}(\tau)\right]=\left[\begin{array}{ll}
c_{1, t}^{\tau} & c_{2, t}^{\tau}
\end{array}\right]\left[\begin{array}{c}
1 \\
i_{t}(\tau)
\end{array}\right]+\left[\varepsilon_{t}\right]} \\
& {\left[\begin{array}{l}
c_{1, t}^{\tau} \\
c_{2, t}^{\tau}
\end{array}\right]=\left[\begin{array}{ll}
1 & 0 \\
0 & 1
\end{array}\right]\left[\begin{array}{l}
c_{1, t-1}^{\tau} \\
c_{2, t-1}^{\tau}
\end{array}\right]+\left[\begin{array}{l}
\eta_{t} \\
\xi_{t}
\end{array}\right]}
\end{aligned}
$$

De forma concisa, o objetivo do filtro de Kalman é obter recursivamente a distribuição condicional dos estados, com observações e modelo dados. Considerando que as variâncias das inovações não são conhecidas, o procedimento tem início na sua estimação. Primeiro, através de um procedimento numérico que garante positividade, as variâncias das inovações são encontradas através da maximização da verossimilhança. 
Desta forma, além das variâncias das inovações, obtém-se também o vetor dos estados filtrados e sua respectiva matriz de covariância filtrada. Após a aplicação do filtro de Kalman, passa-se o filtro para trás, para realizar o alisamento do vetor de estados e da matriz de covariância, encerrando, então, o procedimento de estimação. ${ }^{4}$

\section{Dados}

A base de dados empregada consiste das séries mensais de taxas de juros reais e nominais, para o período de janeiro de 2005 a janeiro de 2010, obtidos na ANBIMA - a Associação Brasileira das Entidades dos Mercados Financeiro e de Capitais. ${ }^{5}$ A estrutura a termo das taxas de juros nominais é extraída a partir dos yields ${ }^{6}$ das NTN-Fs e LTNs, emitidas pelo Tesouro nacional. $\mathrm{O}$ valor de face de uma NTN-F é de R $\$ 1.000,00$, com pagamento de cupons (juros) semestrais de $\mathrm{R} \$ 48,81$. Já a LTN é um título zero-cupom com valor de face de $\mathrm{R} \$ 1.000,00$. Uma vez que trabalhamos com maturidades fixas, empregamos o modelo de Svensson (1994) para ajustar a curva de juros para as maturidades desejadas.

A estrutura a termo das taxas de juros reais também é construída através do modelo de Svensson (1994). Entretanto, a curva é ajustada usando NTN-Bs - a principal classe de títulos indexados à inflação emitidos pelo Tesouro nacional. O yield de uma NTN-B é determinado pelo IPCA, o índice de preços ao consumidor adotado no regime de metas para a inflação do Banco Central do Brasil. Vale ressaltar que as NTN-Bs não apresentam o problema de lag na indexação, como ocorre no mercado de TIPS, uma vez que o juro é pago com base no nível corrente do IPCA, disponível com defasagem máxima de 15 dias. Embora as NTN-Bs sejam emitidas desde 2001, iniciamos nossa base de dados em janeiro de 2005, para evitar problemas de liquidez no mercado do título neste período.

\footnotetext{
${ }^{4}$ Para detalhes sobre estimação por filtro de Kalman ver Durbin e Koopman (2001) e Kim e Nelson (2000).

5 Para mais informações sobre a ANBIMA, acessar http://www.andima.com.br.

6 Também chamado de Taxa Interna de Retorno (TIR) do título.
} 
A estimação de uma estrutura a termo de títulos zero-cupom indexados à inflação e correspondentes BEIRs para o Brasil apresentam duas vantagens relativas às medidas discutidas no artigo. Em primeiro lugar, permite o cálculo de séries de tempo de taxas de juros reais e BEIRs com maturidades fixas, o que é particularmente útil quando se analisa sua evolução sobre um período de tempo relativamente longo. As maturidades dos yields observados e taxas dos títulos existentes não são constantes, porém, declinam ao longo da vida do título, o que pode dificultar a interpretação dos resultados. Segundo, o cálculo de taxas zero-cupom permite que potenciais distorções relacionadas às diferentes durations dos títulos usados no cálculo das BEIRs sejam evitadas. Tais distorções estão relacionadas às diferenças nas estruturas dos fluxos de caixa dos títulos indexados à inflação e títulos nominais. ${ }^{7}$

Ainda assim, a estimação de tais estruturas a termo para o Brasil na conjuntura atual exige a solução de alguns problemas técnicos relacionados ao pequeno número de títulos indexados à inflação, particularmente para as maturidades mais curtas. Apesar do significativo crescimento do mercado de títulos indexados à inflação nos anos recentes, ainda existem algumas limitações a esse respeito. ${ }^{8}$ Conforme mencionado anteriormente, para estimar curvas de juros nominais e reais comparáveis para o mercado brasileiro, empregamos a abordagem paramétrica proposta por Svensson (1994). ${ }^{9}$ Este método assume que a taxa de juros zero-cupom para a maturidade $\tau, \mathcal{Y}(\tau)$, é especificada pela seguinte forma funcional:

7 Duration (Macaulay) é definida como a maturidade média ponderada pelo fluxo de caixa do título. Os pesos são dados pelos valores presentes de cada pagamento como uma proporção do valor presente total dos fluxos de caixa.

8 BEIRs zero-cupom com maturidades fixas podem ser construídas subtraindo taxas reais zero-cupom das taxas nominais zero-cupom de mesma maturidade. Assim, o problema de computar BEIRs zero-cupom com maturidades constantes é basicamente um problema de estimar curvas de juros nominais e reais comparáveis.

9 A literatura sobre estimação de curvas de juros propõe uma variedade de métodos, os quais podem ser divididos em paramétricos e não paramétricos. No caso das abordagens paramétricas, são assumidas formas funcionais parametrizadas parcimoniosamente da curva de juros, e os parâmetros dessas funções são escolhidos para maximizar o ajuste dos preços dos títulos observados. As abordagens não paramétricas são mais flexíveis para ajustar aos preços dos títulos observados, porém, um bom ajuste aos preços observados implica em potencial risco de over-fitting, o qual pode ser especialmente importante para o caso da curva de juros real brasileira, em função de existir um número relativamente pequeno de títulos indexados à inflação. Para mais detalhes sobre ajuste da curva de juros brasileira com modelos paramétricos e não paramétricos, ver Caldeira (2011). 


$$
y(\tau)=\beta_{1}+\beta_{2}\left(\frac{1-e^{\lambda_{1} \tau}}{e^{\lambda_{1} \tau}}\right)+\beta_{3}\left(\frac{1-e^{\lambda_{1} \tau}}{e^{\lambda_{1} \tau}}-e^{\lambda_{1} \tau}\right)+\beta_{4}\left(\frac{1-e^{\lambda_{2} \tau}}{e^{\lambda_{2} \tau}}-e^{\lambda_{2} \tau}\right)
$$

Os parâmetros $\beta_{1}, \beta_{2}, \beta_{3}, \beta_{4}$ e $\lambda$ podem ser estimados através da minimização da diferença entre os preços dos títulos implícitos na forma funcional e preços dos títulos observados. Esta mesma metodologia é aplicada para estimar a curva de juros nominal e real comparáveis, gerando, por conseguinte, as BEIRs com maturidades fixas.

O mercado brasileiro de títulos indexados à inflação é um dos maiores do mundo, com mais de US\$200 bilhões de NTN-Bs em circulação. A maturidade média das NTN-Bs é de, aproximadamente, seis anos. O mercado brasileiro de títulos de renda fixa também é significativo. As LTNs e NTN-Fs têm em torno de US\$ 155 bilhões e US\$ 126 bilhões em circulação, com maturidades médias de 12 meses e 30 meses respectivamente. ${ }^{10}$

Tabela 1 - Estatísticas descritivas

\begin{tabular}{cccccccccc}
\hline $\begin{array}{c}\text { Maturidades } \\
\text { (Meses) }\end{array}$ & Média & $\begin{array}{c}\text { Desvio } \\
\text { Padrão }\end{array}$ & Máximo & Mínimo & Assimetria & Curtose & Jarque-Bera & Correlação & ACF1 \\
\hline $\mathbf{3}$ & $4,96 \%$ & $1,25 \%$ & $10,39 \%$ & $3,20 \%$ & 2,07 & 3,31 & 5,13 & 0,32 & 0,67 \\
$\mathbf{6}$ & $5,06 \%$ & $1,64 \%$ & $11,67 \%$ & $2,68 \%$ & 2,17 & 5,23 & 23,21 & 0,30 & 0,76 \\
$\mathbf{9}$ & $4,92 \%$ & $1,54 \%$ & $10,92 \%$ & $2,93 \%$ & 2,06 & 4,81 & 22,49 & 0,35 & 0,78 \\
$\mathbf{1 2}$ & $4,80 \%$ & $1,38 \%$ & $10,12 \%$ & $3,20 \%$ & 2,03 & 5,17 & 32,58 & 0,36 & 0,79 \\
$\mathbf{1 5}$ & $4,75 \%$ & $1,26 \%$ & $9,48 \%$ & $3,23 \%$ & 1,92 & 5,37 & 30,05 & 0,37 & 0,79 \\
$\mathbf{1 8}$ & $4,76 \%$ & $1,19 \%$ & $9,01 \%$ & $3,22 \%$ & 1,77 & 5,27 & 27,52 & 0,37 & 0,80 \\
$\mathbf{2 4}$ & $4,87 \%$ & $1,12 \%$ & $8,38 \%$ & $3,25 \%$ & 1,38 & 4,42 & 20,31 & 0,36 & 0,80 \\
$\mathbf{3 0}$ & $4,95 \%$ & $1,14 \%$ & $8,00 \%$ & $3,32 \%$ & 1,12 & 3,90 & 9,72 & 0,35 & 0,84 \\
$\mathbf{3 6}$ & $5,00 \%$ & $1,20 \%$ & $7,78 \%$ & $3,39 \%$ & 1,00 & 3,73 & 6,37 & 0,31 & 0,85 \\
IPCA & $4,89 \%$ & $2,77 \%$ & $10,95 \%$ & $-2,49 \%$ & 0,01 & 2,97 & 0,04 & 1,00 & 0,55 \\
& & & & & & & & & \\
\hline Nível & $6,83 \%$ & $1,17 \%$ & $9,76 \%$ & $5,50 \%$ & 1,20 & 4,39 & 17,11 & $-0,04$ & 0,90 \\
Inclinação & $0,17 \%$ & $3,27 \%$ & $9,69 \%$ & $-6,82 \%$ & 0,10 & 3,16 & 0,45 & 0,12 & 0,43 \\
\hline
\end{tabular}

A Tabela 1 apresenta algumas estatísticas descritivas das BEIRs e do IPCA. A média do IPCA para todos os horizontes fica em torno de $4,5 \%$. Tanto as BEIRs quanto a inflação realizada são leptocúrticas,

${ }^{10}$ Estes dados são de dezembro de 2010. Para mais informações sobre o mercado de títulos do Tesouro, ver no site do Banco Central do Brasil, http://www.bcb.gov.br. 
com assimetria positiva, i.e., caudas longas à direita. A estatística Jarque-Bera indica que as BEIRs para horizontes de 3 e 6 meses, assim como IPCA, parecem ser normalmente distribuídos. Isto é um indício de que as BEIRs podem melhor explicar a inflação realizada para horizontes mais curtos. Este indício será confirmado no exercício empírico conduzido na seção 4. Os coeficientes de correlação entre as BEIRs e a inflação realizada são positivos para horizontes de 3, 6 e 12 meses, tornando-se negativos para horizontes de 18, 24 e 36 meses.

A Figura 1, abaixo, descreve a evolução, ao longo do tempo, das BEIRs e taxas de variação no IPCA, para o período que se inicia em janeiro de 2005 até janeiro de 2011. É importante notar que a estrutura a termo das BEIRs é quase sempre positivamente inclinada. Contudo, BEIRs e IPCA não exibem tendência.

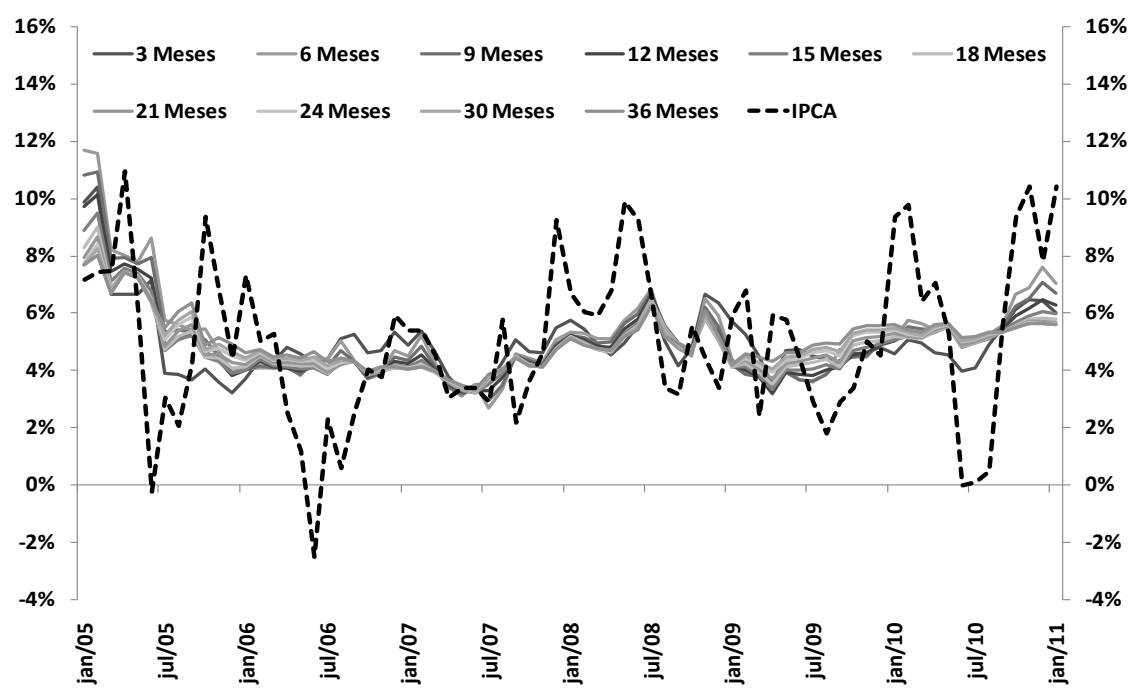

Figura 1 - Evolução BEIRs e IPCA

\section{Estimação e Resultados}

Após estimar a curva de juros nominal e a curva de juros real, comparáveis com maturidades fixas, através do modelo de Svensson (1994) e metodologia explicitada na seção anterior, as BEIRs foram 
geradas e o sistema representado pelas Equações em (4) estimado. O sistema foi estimado pelo filtro de Kalman, com inicializações difusas, para permitir que seus coeficientes variem ao longo do tempo. Assim, não estamos impondo qualquer tipo de restrição na compensação pelo risco incorrido e no coeficiente associado à inflação implícita. Como forma de avaliar a robustez dos resultados obtidos, estimamos uma versão das Equações do sistema (4) sem coeficientes variantes no tempo, dado pela Equação (3), por mínimos quadrados ordinários.

Tabela 2 - Estimativas por filtro de Kalman e mínimos quadrados ordinários

\begin{tabular}{|c|c|c|c|c|c|c|c|c|c|}
\hline \multicolumn{10}{|c|}{ Espaço de Estado - Filtro de Kalman } \\
\hline & 3 & 6 & 9 & 12 & 15 & 18 & 24 & 30 & 36 \\
\hline$c 1$ & $0.0341^{* *}$ & $0.0380^{* *}$ & $0.0414^{* *}$ & $0.0363^{* *}$ & $0.0546^{\star \star}$ & $0.0522^{\star *}$ & $0.0532^{\star *}$ & $0.0519^{* *}$ & $0.0535^{\star *}$ \\
\hline c2 & $0.6331^{* *}$ & $0.2002^{* *}$ & $0.1561^{\star \star}$ & 0,2665 & 0,0344 & $-0.0161^{\star \star}$ & $-0.0468^{* *}$ & $-0.0396^{\star *}$ & $-0.0568^{\star \star}$ \\
\hline \multicolumn{10}{|c|}{ Mínimos Quadrados Ordinários } \\
\hline & 3 & 6 & 9 & 12 & 15 & 18 & 24 & 30 & 36 \\
\hline$c 1$ & $0.0203^{\star *}$ & $0.0356^{\star *}$ & $0.0384^{* *}$ & $0.0402^{\star *}$ & $0.0465^{\star \star}$ & $0.0531^{\star \star}$ & $0.0590^{\star *}$ & $0.0616^{\star \star}$ & $0.0584^{* *}$ \\
\hline $\mathrm{c} 2$ & $0.5781^{\star *}$ & $0.2311^{*}$ & $0.1792^{*}$ & 0,1462 & 0,0073 & $-0,1397$ & $-0.2444^{* *}$ & $-0.2813^{\star *}$ & $-0.2042^{* *}$ \\
\hline
\end{tabular}

Obs.: * $\mathrm{e}^{* *}$ denotam significância a $5 \%$ e $1 \%$, respectivamente.

A Tabela 2 mostra os coeficientes estimados via filtro de Kalman, no estado final suavizado, e por mínimos quadrados ordinários. De forma geral, os resultados obtidos são semelhantes para todas as maturidades consideradas, tanto em magnitude dos coeficientes estimados, quanto em significância estatística de tais coeficientes. Considerando os resultados obtidos pela estimação da representação em espaço de estado, os coeficientes associados às BEIRs se mostraram positivos e estatisticamente significativos para 3, 6 e 9 meses. Isto significa que, para estas maturidades, a BEIR traz informações relevantes a respeito da inflação futura.

Para 12 e 15 meses, os coeficientes associados à BEIR não se mostraram significativos, enquanto para 18, 24, 30 e 36 meses os coeficientes, embora significativos, mostraram-se negativos. Esta relação negativa para maturidades mais longas, segundo Vicente e Guillen (2010), implica que a hipótese de expectativas não se aplica no longo prazo e que, assim, a relação imposta pela Equação (3) pode não ser adequada para explicitar a relação existente entre BEIR e inflação. Uma possível solução para este problema, segundo os autores, seria relaxar a hipótese de constância do prêmio pelo risco. O relaxamen- 
to desta hipótese já foi considerado no sistema (4), estimado pelo filtro de Kalman e, ainda assim, os coeficientes associados às BEIRs permaneceram negativos para 18, 24, 30 e 36 meses. Isto significa que a relação entre BEIR e inflação pode ser altamente complexa.

A constante, que representa a compensação pelo risco incorrido, mostrou-se estatisticamente diferente de zero - embora muito próxima deste valor - para todas as maturidades consideradas. Vale ressaltar, no entanto, que, quando estimado pelo filtro de Kalman, o sistema (4) gerou compensação pelo risco variante no tempo para algumas maturidades, tais como 15, 18, 24, 30 e 36 meses.

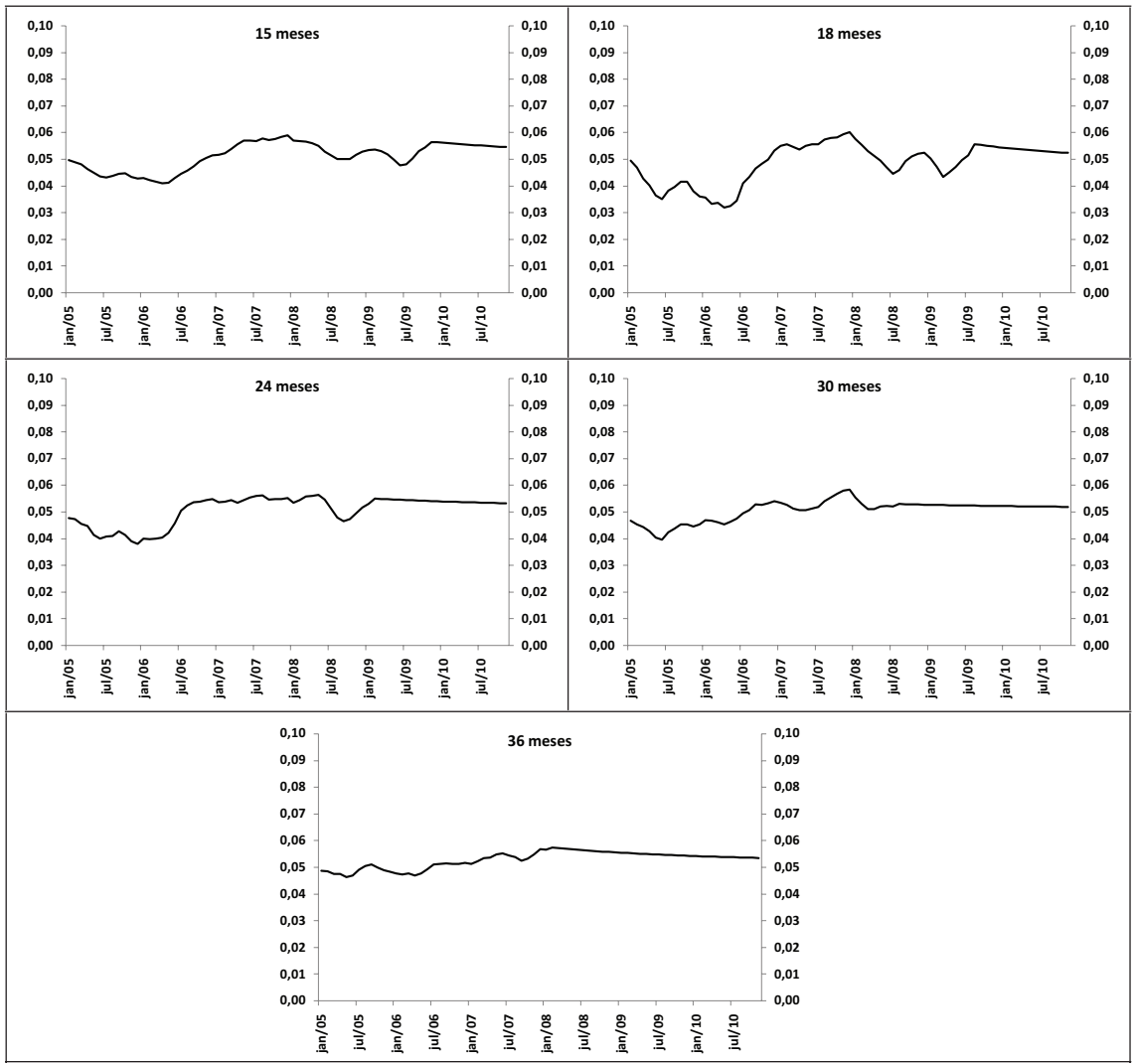

Figura 2 - Compensação pelo risco - estados suavizados 
Uma vez que para quase todas as maturidades consideradas a BEIR traz informação a respeito da inflação futura, torna-se interessante avaliar se ela pode ser considerada um estimador não viesado para a inflação. Para que isto seja verdadeiro, é necessário que, conjuntamente, $c_{1}^{\tau}=0$ e $c_{2}^{\tau}=1$. Realizamos, então, um teste $\mathrm{F}$ para avaliar a validade desta hipótese para cada uma das maturidades.

Tabela 3 - Estatísticas F para $c_{1}^{\tau}=0$ e $c_{2}^{\tau}=1$

\begin{tabular}{cccccccccc}
\hline \multicolumn{10}{c}{ Estatística F } \\
\hline & 3 & 6 & 9 & 12 & 15 & 18 & 24 & 30 & 36 \\
$c 1=0$ & $0.1230^{* *}$ & 0,0000 & 0,0000 & 0,0000 & 0,0000 & 0,0000 & 0,0000 & 0,0000 & 0,0000 \\
$c 2=1$ & & & & & & & & & \\
\hline
\end{tabular}

Obs.: * $\mathrm{e}^{* *}$ denotam significância a $5 \%$ e $1 \%$, respectivamente.

De acordo com a Tabela 3, é possível concluir que a BEIR pode ser considerada um estimador não viesado para a inflação somente para a maturidade de três meses. Para as demais maturidades - 6, 9, 12, 15, 18, 24, 30 e 36 meses - , quando a BEIR traz informação relevante a respeito da inflação, ela o faz de forma viesada.

A Figura 3 apresenta a evolução ao longo do tempo dos coeficientes associados à inflação implícita, obtidos através da estimação do sistema (4) por filtro de Kalman. A maturidade de 15 meses é a única para a qual o coeficiente varia ao longo do tempo, ${ }^{11}$ já para as demais os coeficientes se mantêm relativamente estáveis, condição necessária para a qualidade das previsões.

${ }^{11} \mathrm{O}$ coeficiente associado à inflação implícita apresentou variação ao longo do tempo para as maturidades de 12 e 15 meses, exatamente as duas maturidades em que o coeficiente não se mostrou estatisticamente significativo. Para poupar espaço apresentamos as figures apenas referentes às maturidades $15,18,24,30$ e 36 meses, que foram as que apresentaram compensação pelo risco variante ao longo do tempo. Os gráficos referentes às demais maturidades estão disponíveis com os autores e podem ser fornecidos mediante solicitação. 


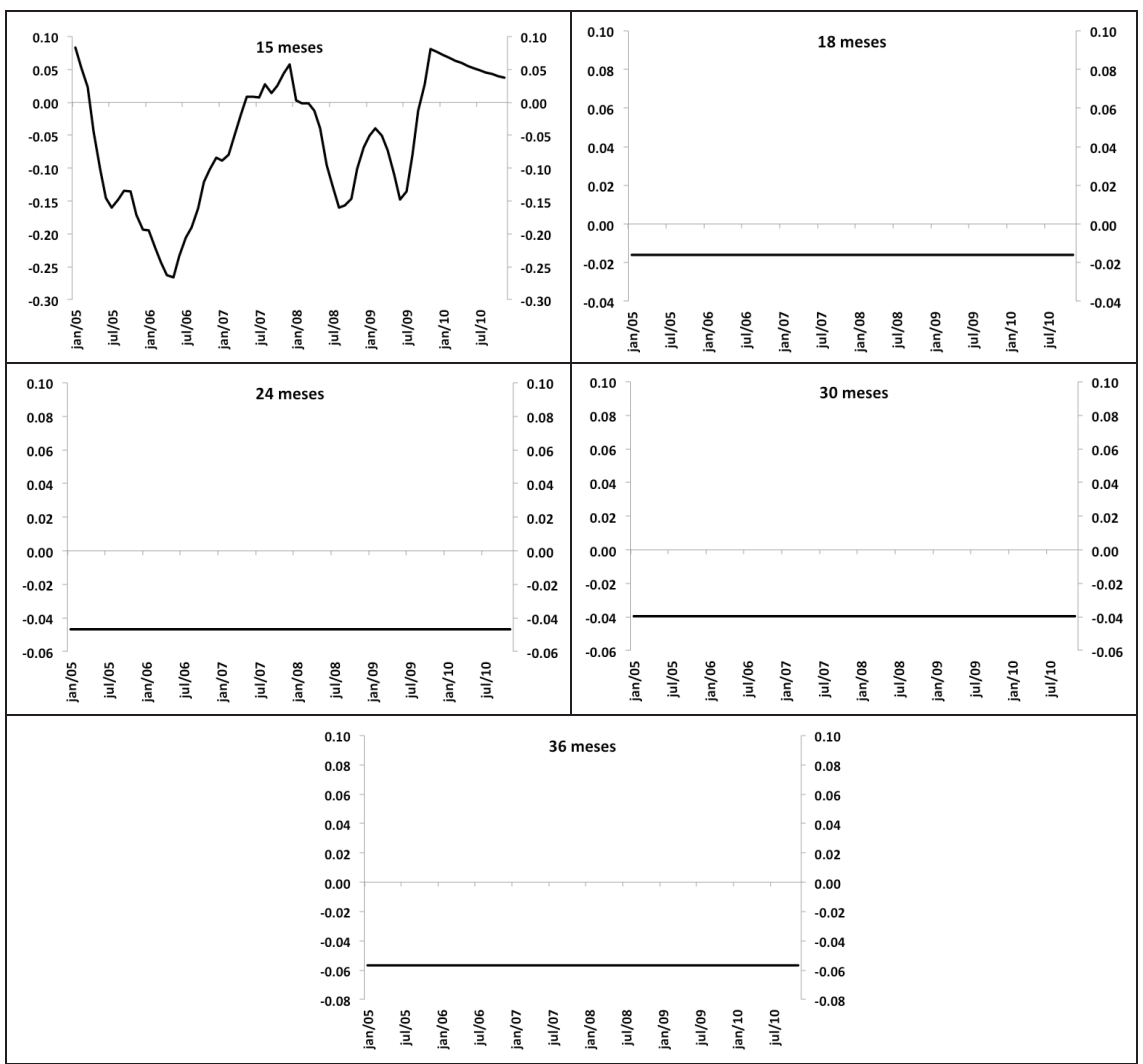

Figura 3 - Coeficiente associado à inflação implícita - estados suavizados

\section{O Poder Preditivo da BEIR}

Mostramos, na seção anterior, que a BEIR traz informação relevante a respeito da inflação futura e que pode ser considerada, inclusive, um estimador não viesado da inflação para maturidade de três meses. A extensão natural deste resultado é exatamente o objetivo secundário do presente trabalho: determinar o poder preditivo da BEIR frente a outras medidas de expectativas de inflação, tais como as constantes nos Relatórios Focus e as extraídas dos modelos autorregressivos - ou, simplesmente VAR - do Banco Central do Brasil.

A escolha das expectativas de inflação do Relatório Focus pode ser facilmente justificada pela sua disponibilidade, por se tratar de publicação de livre acesso a qualquer cidadão - brasileiro ou não -, 
abrangência, pois apresenta resultado de pesquisa de expectativas com cerca de 90 bancos e empresas não financeiras, e publicação semanal, com os resultados das pesquisas realizadas diariamente. ${ }^{12}$

Especificamente para o propósito de nosso trabalho, foi considerada a mediana das expectativas das instituições Top5 de médio prazo para o IPCA. As instituições Top5 são as cinco instituições com as melhores previsões entre aquelas participantes da pesquisa para o Relatório Focus. A escolha das instituições melhores classificadas no ranking de médio prazo, ao invés do ranking de curto prazo, se deve ao horizonte de previsão considerado para a classificação que, para o curto prazo, é somente de um mês, enquanto para o ranking de médio prazo são considerados quatro meses, sinalizando modelos mais consistentes para horizontes mais longos. ${ }^{13}$

O uso dos modelos VAR para este exercício também pode ser facilmente justificado: o Banco Central do Brasil, assim como grande parte de seus pares internacionais, utiliza em larga escala os modelos VAR para análise e previsão de inflação. No caso da autoridade monetária brasileira, os modelos VAR foram introduzidos ao processo de análise logo após a implementação do sistema de metas para a inflação, em junho de 1999. Estes modelos, naturalmente, foram revisados ao longo dos últimos anos, mas ainda constituem insumo importante para o processo decisório do Comitê de Política Monetária do $\mathrm{BCB}$, doravante COPOM. Além disso, os modelos VAR impõem poucas restrições à estrutura da economia. Estas escolhas se resumem, basicamente, à escolha das variáveis e das defasagens. Todo o resto é determinado pelo próprio modelo. Esta é uma característica desejável em um tipo de exercício como o proposto nesta seção, pois reduz o grau de subjetividade da análise.

Os modelos VAR do BCB, basicamente, estão divididos em dois grandes grupos: modelos com fundamentação econômica e modelos puramente estatísticos. Por não possuir restrição econômica - de curto ou longo prazo -, os modelos VAR estatísticos não foram considerados na nossa análise. Entre os modelos com fundamentação econômica foram considerados apenas aqueles em base mensal, para permitir o comparativo com as previsões da BEIR, e estimados de

\footnotetext{
12 Para mais detalhes sobre o Relatório Focus, ver http://www.bcb.gov.br/?INVEST.

${ }^{13}$ Para mais detalhes a respeito dos rankings Top5 do Banco Central do Brasil, incluindo as classificações das instituições e metodologia para o ranking, ver http://www.bcb.gov.br/?FOCUSTOP5.
} 
forma tradicional, não bayesiana. A rigor, portanto, estimamos apenas três modelos VAR, listados na Tabela 4, abaixo.

Tabela 4 - Modelos VAR Mensais Tradicionais do BCB

\begin{tabular}{cccc}
\hline Modelo & Variáveis Endógenas & Ajuste Sazonal & Defasagens \\
\hline VAR I & Preços, Taxa de Câmbio e Juros Reais & sim & 2 \\
VAR II & Preços, Taxa de Câmbio, Juros Nominais, Produção Industrial e Moeda & sim & 6 \\
VAR III & Preços, Taxa de Câmbio e Produção Industrial & não & 1 \\
\hline
\end{tabular}

Primeiramente, é importante ressaltar que os modelos VAR aqui estimados consideram a variação do IPCA, e não dos preços livres e monitorados, como ocorre com os modelos do BCB. O intuito desta alteração é evitar a introdução de subjetividade na análise, já que a alternativa seria realizar algo semelhante ao praticado pelo $\mathrm{BCB}$, que gera a previsão para os preços monitorados, basicamente, em duas etapas: prevê, em um primeiro momento, os principais itens monitorados de forma individual - em uma etapa chamada de previsão item a item - e depois utiliza um modelo econométrico - o chamado modelo de determinação endógena - para os itens restantes, menos representativos.

Além do IPCA, os modelos VAR utilizam: a taxa de câmbio comercial (R\$/US\$), PTAX, final do período, venda; a taxa Selic (\% a.a.), como juros nominais; a taxa Selic (\% a.a.) descontada da inflação medida pelo IGP-DI, como juros reais; a produção industrial, do IBGE, com ajuste sazonal; e, por fim, Ml, como o agregado monetário. A estimação ocorreu com janelas móveis, com amostra iniciando em janeiro de 2000. Adicionalmente, foi considerada no exercício a média das previsões dos modelos VAR. O resultado do comparativo dos erros de previsão, para as diferentes maturidades e modelos, a partir da raiz quadrada do erro quadrático médio, é apresentado na tabela a seguir.

Tabela 5 - Comparativo dos erros de previsão

\begin{tabular}{cccccccccc}
\hline \multicolumn{10}{c}{ Raiz Quadrada do Erro Quadrático Médio } \\
\hline & $\mathbf{3}$ & $\mathbf{6}$ & $\mathbf{9}$ & $\mathbf{1 2}$ & $\mathbf{1 5}$ & $\mathbf{1 8}$ & $\mathbf{2 4}$ & $\mathbf{3 0}$ & $\mathbf{3 6}$ \\
BEIR & 2,18 & 1,93 & 1,66 & 1,51 & 1,54 & 1,55 & 1,50 & 1,50 & 1,46 \\
VAR I & 3,56 & 3,17 & 3,22 & 3,32 & 3,43 & 3,49 & 3,52 & 3,34 & 3,34 \\
VAR II & 4,08 & 3,93 & 3,32 & 2,96 & 2,88 & 2,91 & 3,08 & 3,42 & 3,54 \\
VAR III & 2,85 & 2,97 & 2,96 & 3,02 & 3,19 & 3,34 & 3,37 & 3,44 & 3,51 \\
Média VARs & 2,74 & 2,73 & 2,58 & 2,55 & 2,58 & 2,58 & 2,48 & 2,35 & 2,29 \\
Top5s & 1,97 & 1,44 & 1,26 & 1,23 & - & - & - & - & - \\
\hline
\end{tabular}


A partir da Tabela 5, é possível concluir que os Top5 de médio prazo apresentam os menores erros de previsão da inflação para as maturidades consideradas. As previsões dos Top5, para a maior parte da amostra, foram realizadas apenas para os 12 meses seguintes - por uma limitação então existente no sistema de expectativas do $\mathrm{BCB}$-, o que limita a comparação para as maturidades de 15, 18, 24, 30 e 36 meses. Para estas maturidades mais longas, a BEIR mostra maior acurácia. Os modelos VAR considerados apresentaram projeções menos precisas que a BEIR e os Top5. O melhor resultado para os modelos VAR foi gerado pela média entre VAR I, VAR II e VAR III.

Este é um resultado importante, pois sugere que a BEIR supera os modelos VAR considerados - individualmente ou a média destes modelos - para previsão da inflação, quando medida pelo IPCA, em todas as maturidades consideradas. É fato que os Top5 de médio prazo apresentam melhores projeções, mas este é um resultado, até certo ponto, esperado. Isto porque os Top5 utilizam não somente estes e outros modelos VAR, mas também modelos mais complexos e bem-sucedidos, como os VARs estruturais, além dos modelos macroeconômicos estruturais.

\section{Conclusões}

O presente trabalho se propôs a avaliar, para o caso brasileiro, se as informações sobre a inflação implícita extraídas dos títulos de renda fixa trazem informação relevante a respeito da inflação futura, sem partir de hipóteses restritivas. Propusemos uma forma inovadora de estimar esta relação, através do Filtro de Kalman, para permitir que a compensação pelo risco e o poder explicativo da inflação implícita variassem ao longo do tempo.

Os resultados de nossas estimações sugerem que, de forma geral, a BEIR traz informação a respeito da inflação futura. Os coeficientes associados às BEIRs se mostraram positivos e estatisticamente significativos para 3, 6 e 9 meses. Para 12 e 15 meses, os coeficientes associados à BEIR não se mostraram significativos, enquanto para 18 , 24, 30 e 36 meses, os coeficientes, embora significativos, mostraramse negativos. A compensação pelo risco incorrido, representada pela constante, mostrou-se estatisticamente diferente de zero e muito próxima deste valor para todas as maturidades consideradas. 
Esta compensação pelo risco revelou comportamento variante no tempo para algumas maturidades, tais como 15, 18, 24, 30 e 36 meses.

Avaliamos ainda se a BEIR poderia ser considerada um estimador não viesado para a inflação. Para que isto fosse verdadeiro, seria necessário que, conjuntamente, a constante fosse igual a zero e o coeficiente associado à BEIR igual à unidade. Realizamos, então, um teste $\mathrm{F}$ para avaliar a validade desta hipótese para cada uma das maturidades, e concluímos que a BEIR pode ser considerada um estimador não viesado para a inflação somente para a maturidade de três meses.

Por fim, dispusemo-nos a determinar o poder preditivo da BEIR frente a outras medidas de expectativas de inflação, tais como as constantes nos Relatórios Focus e as extraídas dos modelos VAR do Banco Central. Nossas estimativas apontaram que as previsões realizadas com as BEIRs mostraram maior acurácia que aquelas extraídas dos modelos VAR. Estas, por sua vez, mostraram-se menos precisas que as geradas pelos Top5.

\section{Referências}

ANG, A.; BEKAERT, G.; Wei, M. Do Macro Variables, Asset Markets Forecast Inflation Better? Journal of Monetary Economics, 54, 1163-1212, 2007.

BARR, D.G.; CAMPBELL, J.Y. Inflation, real interest rates, and the bond market: a study of UK nominal and index-linked government bond prices. Journal of Monetary Economics, Vol. 39(3), p. 361-383, 1997.

BERNANKE, B. What policy makers can learn from asset prices. Disponível em $<$ http://www.federalreserve.gov/boarddocs/speeches/2004/20040415/default.htm>, 2004.

BREEDON, F. Bond prices and market expectations of inflation. Bank of England, Quarterly Bulletin, Maio, p. 160-165, 1995.

BURASCHI, A.; JILTSOV, A. Inflation Risk Premia and the Expectation Hypothesis, Journal of Financial Economics, 75, 429-490, 2005.

CALDEIRA, J. F. Estimação da Estrutura a Termo da Curva de Juros no Brasil Através de Modelos Paramétricos e Não-Paramétricos. Análise Econômica, 29 (55), 95-122, 2011.

CAMPBELL, J.Y.; SHILLER, R.J.A Scorecard for Indexed Debt. NBER Macroeconomics Annual, pp. 155-197, 1996.

CHEN, R. R.;LIU, B.; CHENG, X. Inflation, Fisher Equation, and the Term Structure of Inflation Risk Premia: Theory and Evidence from TIPS. Journal of Empirical Finance, 17 (4), 702-721, 2010.

D’AMICO, S.; KIM, D. H.; WEI, M. Tips from TIPS: the informational content of Treasury InflationProtected Security Prices, Working paper, Board of Governors of the Federal Reserve System (U.S.) n.19, 2010. 
DEACON, M.; ANDREWS, P. The use and value of index-linked bonds. The Financier, Vol. 3, 1996.

Durbin, J.; Koopman, S. (2001).Time Series Analysis by State Space Methods. Oxford, New York.

EMMONS, W.R. The information content of Treasury Inflation-Indexed Securities. Federal Reserve Bank of St Louis, Review, Novembro/Dezembro, p. 25-37, 2000.

EVANS, M. Real Rates, Expected Inflation, and Inflation Risk Premia. The Journal of Finance, 53(1), $187-218,1998$.

GARCIA, J.A.; VAN RIXTEL, A. Inflation-linked bonds from a central bank perspective. European Central Bank, Occasional Paper Series, N. 62, 2007.

GRISHCHENKO, O.; HUANG, J. Inflation Risk Premium: Evidence from the TIPS market. Working Paper, Disponível em<http://ssrn.com/abstract=1364254>, 2010.

HAUBRICH, J.; PENNACCHI, G.; RITCHKEN, P. 2008, Estimating Real and Nominal Term Structures using Treasury Yields, Inflation Forecasts, and Inflation Swap Rates, Working Paper, Federal Reserve Bank of Cleveland, University of Illinois at Urbana- Champaign and Case Western Reserve University, 2008

HETZEL, R.L. Indexed bonds as an aid to monetary policy. Federal Reserve Bank of Richmond, Economic Review, Janeiro/Fevereiro, p. 13-23, 1992.

HORDAHL, P.; Tristani, O.; D. Vestin, D. The yield curve and macroeconomic dynamics. Economic Journal, 118(533), 1937-70, November 2008.

JARROW, R.; Yildirim, Y. Pricing Treasury Inflation Protected Securities and Relative Derivatives using HJM Model, Journal of Financial and Quantitative Analysis, 38(2), 337-358, 2003.

Kim, C.J.; Nelson, C.R. 1999. State-Space Models with Regime Switching. Cambridge, Massachusetts: MIT Press.

KITAMURA, Y. Indexed bonds and monetary policy: the real interest rate and the expected rate of inflation. Bank of Japan, Monetary and Economic Studies, Vol. 15(1), p. 1-25, 1997.

SVENSSON, L. E. O. 1994 (Sept.). Estimating and Interpreting Forward Interest Rates: Sweden $1992-$ 1994. IMF Working Papers 94/114. International Monetary Fund.

VICENTE, J.V.M.; GUILLEN, O.T.C. Do inflation-linked bonds contain information about future inflation? Banco Central do Brasil, Working Paper Series, N. 214, 2010. 